\title{
Reduction of MLH1 and PMS2 confers temozolomide resistance and is associated with recurrence of glioblastoma
}

\author{
Yoshinari Shinsato ${ }^{1,2}$, Tatsuhiko Furukawa ${ }^{2}$, Shunji Yunoue ${ }^{1}$, Hajime Yonezawa ${ }^{1}$, \\ Kentarou Minami2,3, Yukihiko Nishizawa ${ }^{2,3}$, Ryuji Ikeda ${ }^{3}$, Kohichi Kawahara ${ }^{2}$, \\ Masatatsu Yamamoto ${ }^{2}$, Hirofumi Hirano ${ }^{1}$, Hiroshi Tokimura ${ }^{1}$, and Kazunori Arita ${ }^{1}$ \\ ${ }^{1}$ Department of Neurosurgery, Graduate School of Medical and Dental Sciences Kagoshima University, Kagoshima, Japan; \\ 2 Department of Molecular Oncology, Graduate School of Medical and Dental Sciences Kagoshima University, Kagoshima, \\ Japan; \\ ${ }^{3}$ Department of Clinical Pharmacy and Pharmacology, Graduate School of Medical and Dental Sciences Kagoshima University, \\ Kagoshima, Japan
}

Correspondence to: Tatsuhiko Furukawa, email: furukawa@m3.kufm.kagoshima-u.ac.jp

Keywords: temozolomide, MLH1, PMS2, MutL-alpha, resistance, recurrence, glioblastoma

Received: August 20,2013 Accepted: October 12,2013 Published: October 14, 2013

This is an open-access article distributed under the terms of the Creative Commons Attribution License, which permits unrestricted use, distribution, and reproduction in any medium, provided the original author and source are credited.

ABSTRACT:

Although there is a relationship between DNA repair deficiency and temozolomide (TMZ) resistance in glioblastoma (GBM), it remains unclear which molecule is associated with GBM recurrence. We isolated three TMZ-resistant human GBM cell lines and examined the expression of $0^{6}$-methylguanine-DNA methyltransferase (MGMT) and mismatch repair (MMR) components. We used immunohistochemical analysis to compare MutL homolog 1 (MLH1), postmeiotic segregation increased 2 (PMS2) and MGMT expression in primary and recurrent GBM specimens obtained from GBM patients during TMZ treatment. We found a reduction in MLH1 expression and a subsequent reduction in PMS2 protein levels in TMZ-resistant cells. Furthermore, MLH1 or PMS2 knockdown confered TMZ resistance. In recurrent GBM tumours, the expression of MLH1 and PMS2 was reduced when compared to primary tumours.

\section{INTRODUCTION}

Treatment with the alkylating agent temozolomide (TMZ) has resulted in benefits for patients with glioblastoma (GBM). Nevertheless, almost all GBMs recur and lead to death of the patients [1-3]. Intrinsic or acquired resistance to TMZ, is one of the greatest obstacles in successful GBM treatment, and is thought to be influenced by a variety of mechanisms.

Studies indicate that DNA repair molecule deficiency is linked to the acquisition of TMZ resistance in GBM; however clarification on which molecules are most important in the attainment of TMZ resistance is required. For instance, $\mathrm{O}^{6}$-methylguanine-DNA methyltransferase (MGMT) (ENSG00000170430), which is the best-known cause of TMZ resistance, is only expressed at low levels in many GBMs due to the methylation of its promoter region. Mismatch repair (MMR)-dependent correction of replication errors and responses to DNA damage requires heterodimeric complexes of MutS-alpha (mutS homolog 6; MSH6; ENSG00000116062 / mutS homolog 2; MSH2; ENSG00000095002) and MutL-alpha (MutL homolog 1; MLH1; ENSG00000076242 / postmeiotic segregation increased 2; PMS2; ENSG00000122512). MutS-alpha initially recognizes DNA mismatches, while MutL-alpha identifies the mismatch and subsequently excises the nascent error containing DNA strand [4]. Previous studies have suggested that there is a relationship between deficiencies in these four MMR components and GBM recurrence [5-7]; however it remains unclear which MMR components are most important in influencing the acquisition of TMZ resistance.

Therefore, we established TMZ-resistant cell lines that do not express MGMT from U251 human GBM cells and analysed their TMZ resistance. In addition, we analysed the expression of MLH1, PMS2 and MGMT in primary and recurrent GBM tumour samples obtained from patients with GBM recurrence during TMZ treatment. 


\begin{tabular}{|c|c|c|c|c|c|c|c|}
\hline \multicolumn{7}{|l|}{ Table 1: Sensitivity of TMZ-resistant cells to several methylating reagents } \\
\hline Cells & $\mathrm{U} 251$ & \multicolumn{2}{|c|}{$\mathrm{U} 251 / \mathrm{TMZR} 1$} & $\mathrm{U} 251 / \mathrm{TMZR} 2$ & \multicolumn{2}{c|}{$\mathrm{U} 251 / \mathrm{TMZR} 3$} \\
\hline Reagent & $\mathrm{IC}_{50}$ & $\mathrm{IC}_{50}$ & $\mathrm{RR}$ & $\mathrm{IC}_{50}$ & $\mathrm{RR}$ & $\mathrm{IC}_{50}$ & $\mathrm{RR}$ \\
\hline $\mathrm{TMZ}(\mathrm{mM})$ & $67.6 \pm 11.2$ & $454.1 \pm 30.0$ & $6.7 \pm 0.9^{\mathrm{a}}$ & $871.0 \pm 105.4$ & $12.9 \pm 0.8^{\mathrm{a}}$ & $569.5 \pm 44.7$ & $8.4 \pm 0.8^{\mathrm{a}}$ \\
\hline $\mathrm{MNNG}(\mathrm{ng} / \mathrm{ml})$ & $0.45 \pm 0.02$ & $2.6 \pm 0.04$ & $5.6 \pm 0.3^{\mathrm{a}}$ & $3.5 \pm 0.22$ & $7.7 \pm 0.7^{\mathrm{a}}$ & $1.7 \pm 0.04$ & $3.8 \pm 0.09^{\mathrm{a}}$ \\
\hline $\mathrm{ACNU}(\mathrm{mM})$ & $87.3 \pm 3.8$ & $32.3 \pm 1.75$ & $0.37 \pm 0.01^{\mathrm{a}}$ & $58.1 \pm 4.4$ & $0.7 \pm 0.05^{\mathrm{a}}$ & $111.8 \pm 4.7$ & $1.7 \pm 0.1^{\mathrm{a}}$ \\
\hline $\mathrm{MMS}(\mathrm{mM})$ & $131.5 \pm 9.1$ & $88.4 \pm 4.6$ & $0.7 \pm 0.03^{\mathrm{a}}$ & $206.2 \pm 1.6$ & $1.6 \pm 0.1^{\mathrm{a}}$ & $165.0 \pm 8.9$ & $1.3 \pm 0.1^{\mathrm{a}}$ \\
\hline
\end{tabular}

Cell survival was determined using the MTT assay.

Data are means \pm S.D. of three determinations obtained from triplicate cultures.

a Significantly different $(\mathrm{p}<0.05)$ compared with U251 cells, as determined by Student's $t$ test.

U251, parental glioblastoma cell line; TMZR1-R3, TMZ-resistant U251 cells; TMZ: temozolomide; MNNG: N-methyl-N'-nitro-N-

nitrosoguanidine; ACNU: Nimustine; MMS: methyl methanesulfonate; RR: relative resistance (fold resistance compared to U251 parental cells).

\section{RESULTS}

\section{Generation and analysis of TMZ-resistant cell lines.}

To analyse cellular TMZ resistance mechanisms, we generated TMZ-resistant U251 cell lines (U251/TMZR1, U251/TMZR2 and U251/TMZR3 cells). MTT assays showed that these cells displayed a resistance to TMZ that was increased by 6.7-fold, 12.9-fold and 8.4-fold, respectively, when compared with that of U251 cells. In addition, the TMZ-resistant cells showed cross-resistance to a SN1-type methylating agent, N-Methyl-N ' -nitro-Nnitrosoguanidine (MNNG), which has properties similar to those of TMZ. The cells did not show obvious resistance to other types of methylating agents such as nimustine (ACNU), which is a SN2-type methylating agent, or methyl methanesulfonate (MMS), which, unlike TMZ, does not add methyl groups to the $\mathrm{O}^{6}$ position of guanine nucleotides (Table 1).

\section{MGMT is not involved in the acquisition of resistance to TMZ.}

An increase in cellular MGMT activity is the bestknown mechanism by which cells acquire TMZ resistance $[8,9]$. However, neither MGMT mRNA nor MGMT protein were detectable in the TMZ-resistant cells or the U251 cells (Supplementary Figure S1A, B). The MGMT promoter region has been reported to be methylated and, thereby, inactivated in U251 cells. Therefore, we examined the status of the promoter region of the MGMT gene in U251 and the TMZ-resistant cells by using methylationspecific PCR. This analysis indicated that the promoter region of the MGMT gene in U251 and TMZ-resistant cells was methylated (Supplementary Figure S1C).
G2/M arrest and apoptosis is induced by TMZ in U251 cells but not in U251/TMZR2 cells.

Next, the cell cycle populations of U251 and U251/TMZR2 were analysed to determine whether the decreased sensitivity of the U251/TMZR2 cells to TMZ resulted in a reduction of cell cycle arrest and cell death. After treatment with 800 micro-M TMZ for 120 h, U251 cells were mostly arrested in the G2/M phase of the cell cycle, and there was an increase in the sub- $\mathrm{G}_{1}$ fraction of cells when compared to the control cells. In contrast, TMZ treatment did not alter the cell cycle distribution, or the sub-G $\mathrm{G}_{1}$ fraction of U251/TMZR2 cells when compared to control cells (Supplementary Figure S2A). We then measured caspase-3 activity in U251 and U251/TMZR2 cells after treatment with 800 micro M TMZ for $96 \mathrm{~h}$. Our results showed that caspase-3 activity in U251 cells was $5.9 \pm 0.6-$ fold higher than in U251/TMZR2 cells $(p<0.01)$ (Supplementary Figure S2B). These results demonstrate that TMZ induces MMR mediated G2/M arrest and apoptosis in parental cells, whereas acquired resistance to TMZ protects cells from TMZ-induced G2/M arrest and apoptosis.

\section{Reduction of MLH1 expression and subsequent reduction in PMS2 protein expression is involved in $\mathrm{TMZ}$ resistance.}

DNA alkylating agents such as MNNG and TMZ have been reported to induce MMR, DNA damageinduced G2 checkpoint, and apoptosis [10-14]. To determine whether MMR systems were altered in the TMZ-resistant cells, we compared the expression of the MMR proteins MSH6, MSH2, MLH1, and PMS2 in U251 cells and TMZ-resistant cells. We found that the mRNA and protein expression of MLH1 was consistently lower in the TMZ-resistant cells than in the U251 cells (Fig. 1A, B). Furthermore, the mRNA expression of MLH1 was significantly induced by TMZ in a time dependent manner in U251 cells, whereas only slight TMZ-mediated 

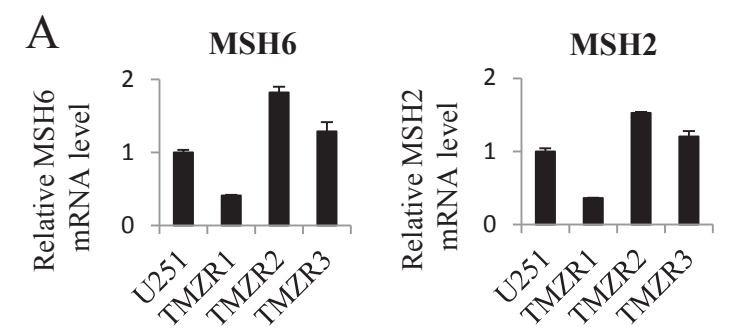

B
MSH6
RD
MSH2
RD
MLH1

B
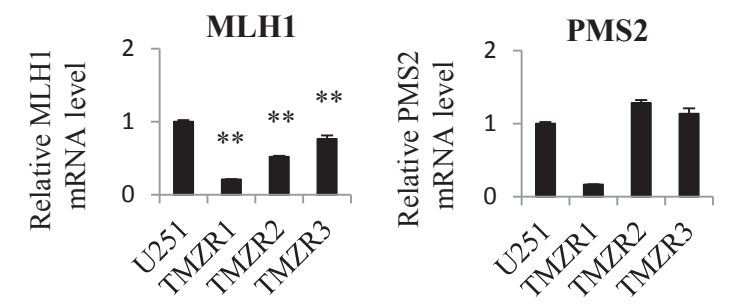

RD

PMS2

$\mathrm{RD}$
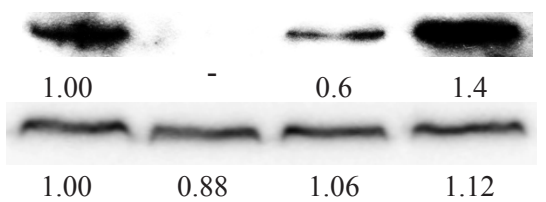

GAPDH
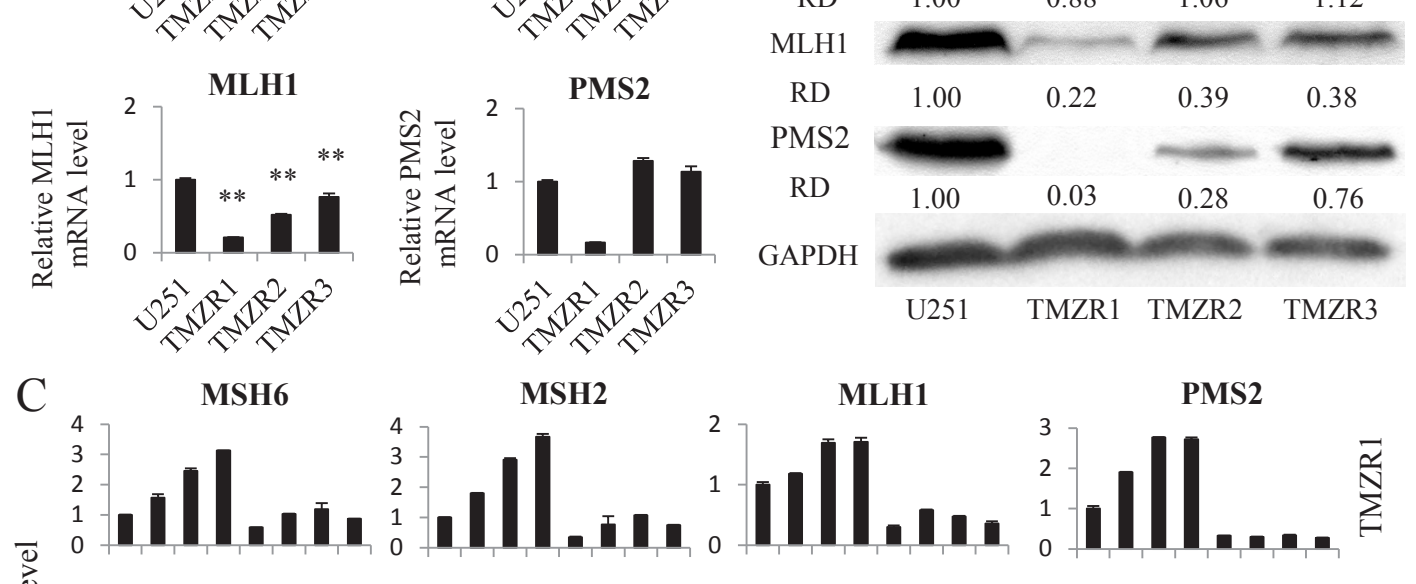

MSH2

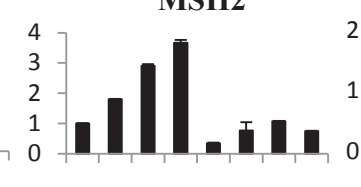

MLH1

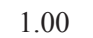

0.22

0.39

0.38

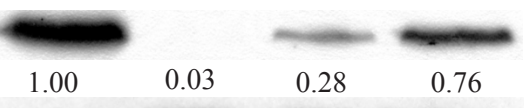

GAPDH
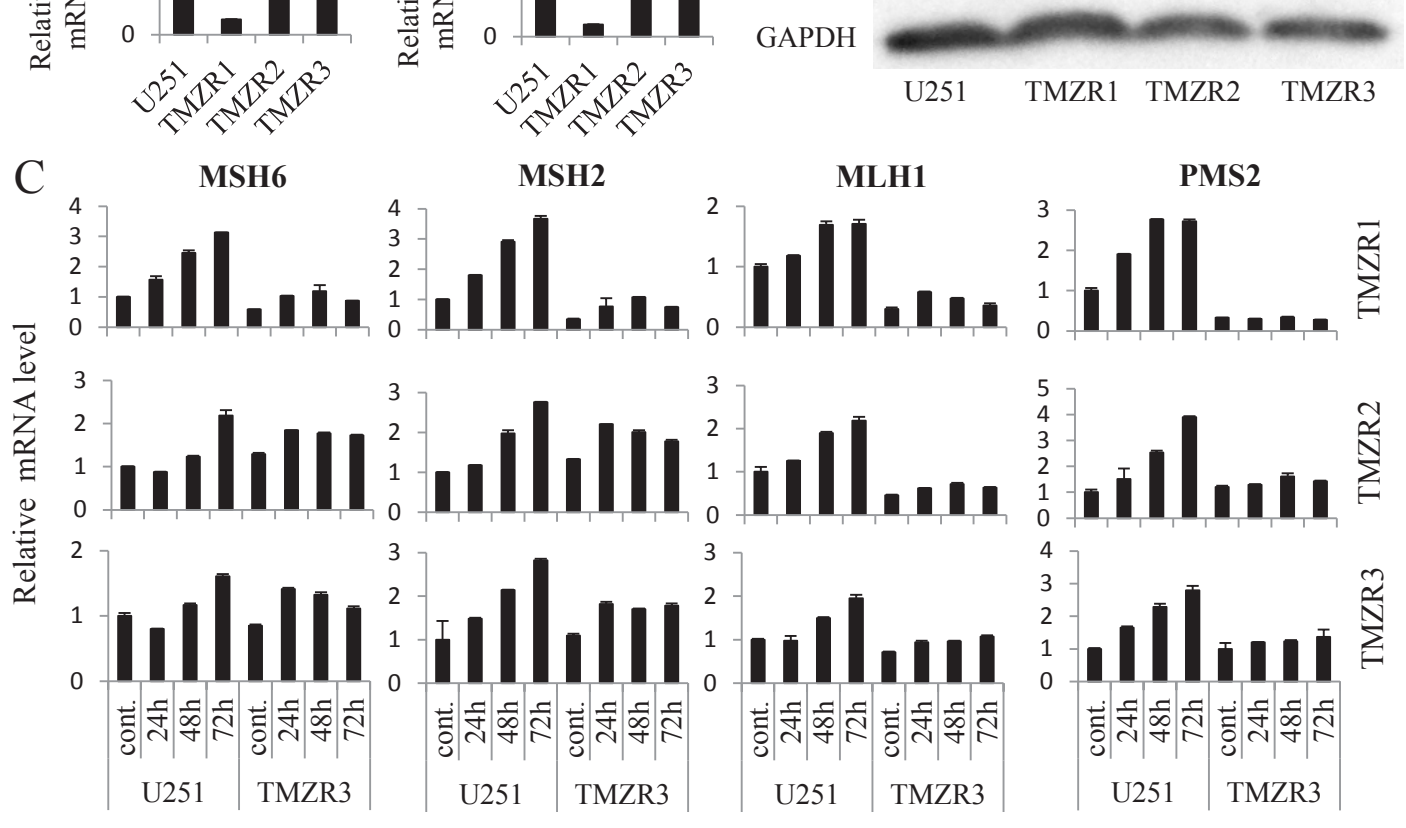

D $\beta$-catenin
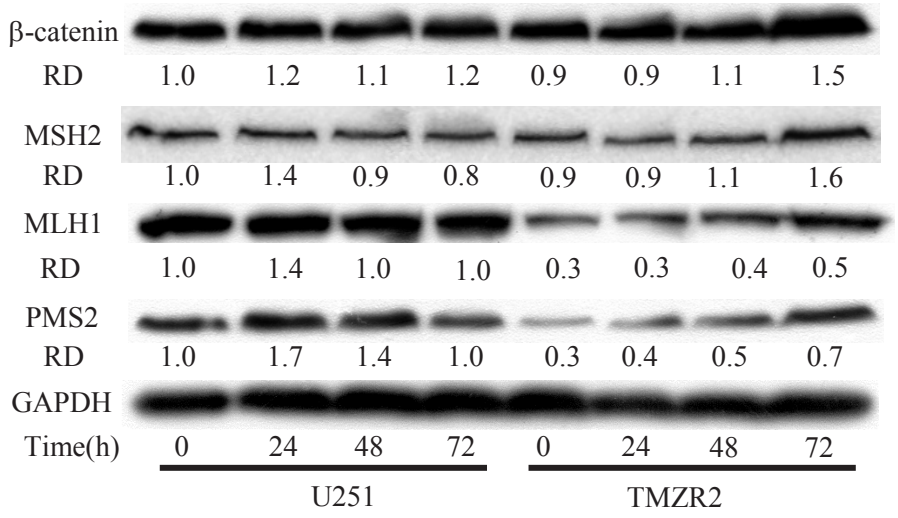

Figure 1: The expression of mismatch repair components in TMZ-resistant cells. (A) The mRNA levels of MSH6, MSH2, MLH1, PMS2 in U251 and TMZ-resistant cells (U251/TMZR1, U251/TMZR2 and U251/TMZR3 cells) was analysed using real-time PCR. GAPDH mRNA expression levels were used to normalize the values obtained for each gene. Columns are representative of triplicate independent experiments and bars represent SD. ${ }^{* *}, P<0.01$, significantly different from the value of U251 cells. (B) The level of MSH6, MSH2, MLH1 and PMS2 protein expression in U251 and TMZ-resistant cells was detected by immunoblotting, as described in the Materials and Methods. GAPDH protein levels were assayed as loading controls. The densities of the individual bands were quantified using Alpha View software, and were normalized to GAPDH in order to obtain the relative densities (RD). (C) Time-dependent changes in MMR gene expression after 400 micro-M TMZ treatment at the indicated times were analysed using real-time PCR. GAPDH mRNA expression was used to normalize the values obtained for the MMR genes. (D) Time-dependent changes in MSH2, MLH1 and PMS2 protein expression after 400 micro-M TMZ treatment at the indicated times were detected by immunoblotting as described in the Materials and Methods. betaCatenin protein levels were used as a negative control. GAPDH protein levels were assayed as loading controls. Densities of the individual bands were quantified using Alpha View software and normalized to GAPDH in order to obtain the relative densities (RD). 
inductions were observed in the three TMZ-resistant cell lines. In addition, the expression of MLH1 protein in TMZ-resistant cells was also lower than that of U251 cells at all time points after TMZ treatment (Fig. 1C, D). Notably, the expression of PMS2 protein was correlated with the expression of MLH1 protein but not to PMS2
mRNA expression levels in these three TMZ-resistant cell lines. In addition, the induction of PMS2 protein and mRNA, as well as MLH1 protein, after TMZ treatment was also lower than that in the parent cells (Fig. 1C, D). These results suggest that the reduction of MLH1 and/or PMS2 is involved in TMZ resistance.
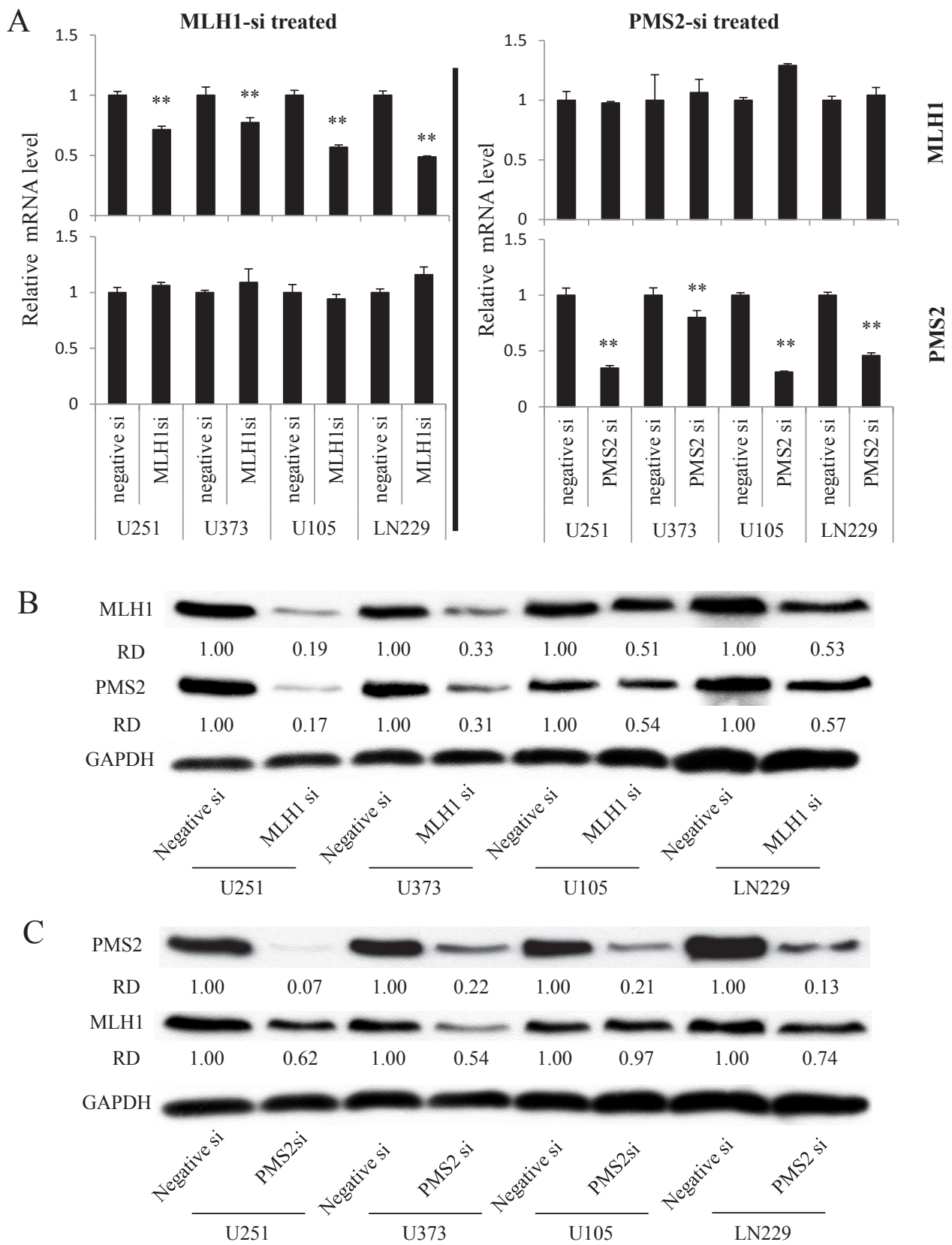

Figure 2: Effect of MLH1 or PMS2-specific siRNA treatment on MLH1 or PMS2 expression in several GBM cell lines. GBM cells were transfected with $37.5 \mathrm{nM}$ negative control (Negative-si), MLH1-specific (MLH-si), or PMS2-specific (PMS2-si) siRNA. (A) At $48 \mathrm{~h}$, the mRNA levels of MLH1 and PMS2 were analysed using real-time PCR. GAPDH mRNA expression was used to normalize the values obtained for MLH1 and PMS2. Columns are representative of triplicate independent experiments, and bars indicate SD. **, $P$ $<0.01$, significantly different from Negative-si treated GBM cells. (B) The protein levels of MLH1 or PMS2 in Negative-si or MLH1-si treated GBM cells were detected by immunoblotting, as described in the Materials and Methods. GAPDH protein levels were assayed as the loading control. The densities of the individual bands were quantified using Alpha View software, and normalized to GAPDH in order to obtain the relative densities (RD). (C) The protein levels of MLH1 or PMS2 in Negative-si or PMS2-si treated GBM cells were detected by immunoblotting, as described in the Materials and Methods. GAPDH protein levels were assayed as the loading control. The densities of individual bands were quantified using Alpha View software and normalized to GAPDH in order to obtain relative densities (RD). 
Table 2: MLH1 and PMS2 expression levels modulate the TMZ sensitivity of several glioma cells.

\begin{tabular}{|c|c|c|c|c|c|c|c|c|}
\hline \multirow{2}{*}{ cells } & \multicolumn{2}{|c|}{$\mathrm{U} 251$} & \multicolumn{2}{c|}{$\mathrm{U} 373$} & \multicolumn{2}{c|}{$\mathrm{U} 105$} & \multicolumn{2}{c|}{ LN229 } \\
\cline { 2 - 9 } & $\mathrm{IC}_{50}{ }^{\mathrm{a}}(\mu \mathrm{M})$ & $\mathrm{RR}$ & $\mathrm{IC}_{50}{ }^{\mathrm{a}}(\mu \mathrm{M})$ & $\mathrm{RR}$ & $\mathrm{IC}_{50}{ }^{\mathrm{a}}(\mu \mathrm{M})$ & $\mathrm{RR}$ & $\mathrm{IC}_{50}{ }^{\mathrm{a}}(\mu \mathrm{M})$ & $\mathrm{RR}$ \\
\hline Negative si & $46.9 \pm 7.3$ & - & $10.7 \pm 1.3$ & - & $116.9 \pm 31.6$ & - & $84.9 \pm 22.8$ & - \\
\hline MLH1 si & $529.3 \pm 25.6$ & $11.4 \pm 1.3^{\mathrm{b}}$ & $125.1 \pm 29.3$ & $11.6 \pm 1.3^{\mathrm{b}}$ & $641.1 \pm 15.6$ & $5.7 \pm 1.5^{\mathrm{b}}$ & $175.2 \pm 19.2^{2}$ & $2.1 \pm 0.4^{\mathrm{b}}$ \\
\hline PMS2 si & $625.6 \pm 49.4$ & $13.4 \pm 1.1^{\mathrm{b}}$ & $131.5 \pm 6.8$ & $12.4 \pm 1.1^{\mathrm{b}}$ & $671.4 \pm 46.0$ & $6.0 \pm 1.2^{\mathrm{b}}$ & $310.0 \pm 87.2$ & $3.7 \pm 0.1^{\mathrm{b}}$ \\
\hline
\end{tabular}

${ }^{a}$ Cell survival was determined using the MTT assay.

${ }^{\mathrm{b}}$ Significantly different $(\mathrm{p}<0.05)$ compared with Negative siRNA-treated cells, as determined by Student's t test.

\section{Diminished MLH1 expression reduces the expression of PMS2 protein and modulates TMZ sensitivity in several GBM cell lines.}

To confirm whether the reduction of MLH1 expression attenuates PMS2 protein and confers TMZ resistance, we tested several GBM cells using MLH1 siRNA knockdown. MLH1-specific siRNA significantly reduced not only the mRNA and protein expression of MLH1 in GBM cells, but also the expression of PMS2 protein (Fig. 2A, B). Notably, knockdown of MLH1 did not affect the expression of PMS2 mRNA (Fig. 2A). In contrast, knockdown of PMS2 by siRNA did not affect the mRNA expression of MLH1, but slightly affected the protein expression of MLH1 (Fig. 2A, C). In addition, MLH1 or PMS2-specific siRNA-treated GBM cells were resistant to TMZ when compared to control siRNA-treated cells (Table 2). These data indicate that MLH1 is involved

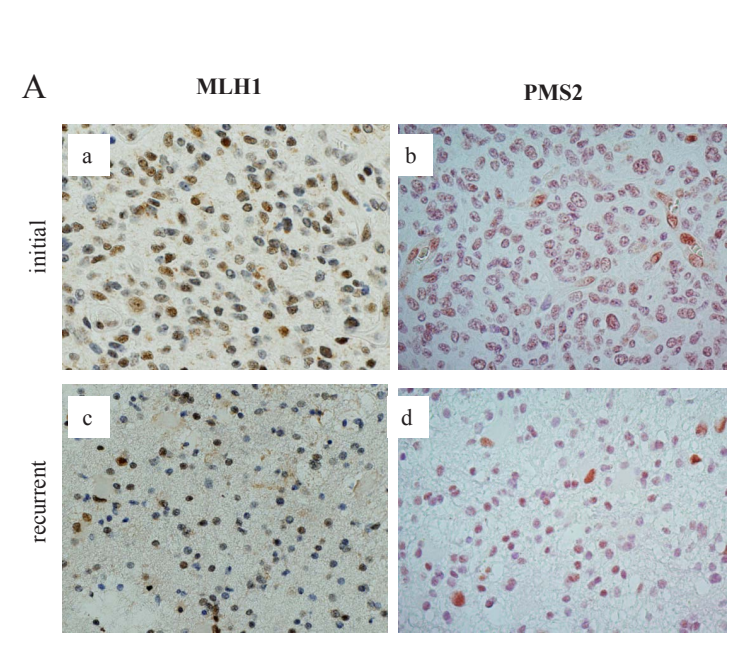

in PMS2 protein stability, and attenuation of MLH1 and PMS2 confers TMZ resistance to GBM cells.

\section{The expression of MLH1 and PMS2 is reduced in recurrent human glioblastomas during administration of TMZ.}

If the reduction of MLH1 and/or PMS2 expression plays an important role in the acquisition of TMZ resistance in vivo as well as in vitro, MLH1 and/or PMS2 attenuation might be involved in the recurrence of GBMs. We therefore evaluated MLH1 and PMS2 expression in a total of 11 clinical GBM and AA specimens using immunohistochemical methods. Immunohistochemical analysis of initial and recurrent tumours of a representative case that was treated with TMZ is shown in Fig. 3A, a-d For all cases, the expressions of MLH1 and PMS2 were significantly decreased in the recurrent specimens
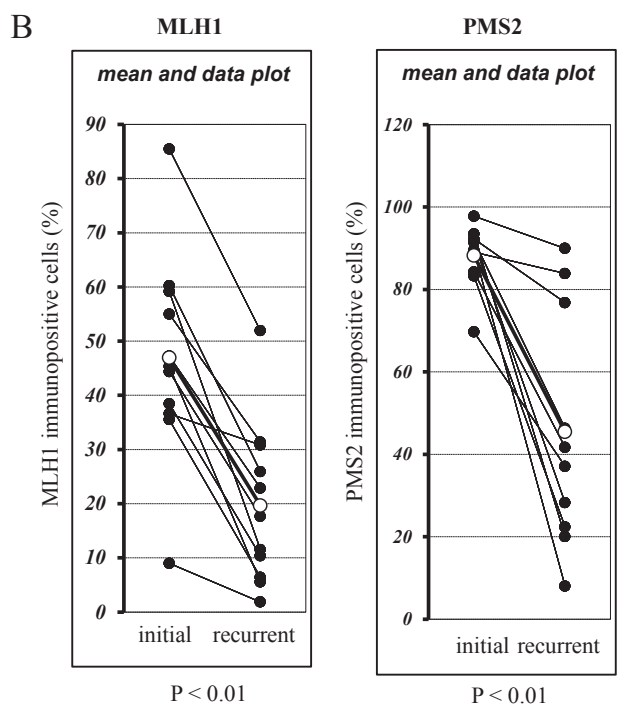

Figure 3: Immunohistochemical analysis of the expression of MLH1 and PMS2 in recurrent human glioblastomas during TMZ treatment. MLH1 and PMS2 protein expression in nine clinical glioblastoma and two anaplastic astrocytoma specimens was analysed by using immunohistochemical analysis, as described in the Materials and Methods. The fraction of MLH1 or PMS2 positive cells in each specimen was determined by dividing the number of MLH1 or PMS2 immunopositive cells by the total cell number in three microscopic fields (magnification $\times 400$ ). (A) Immunohistochemical staining of initial and recurrent tumours of one representative case. (a) MLH1 staining in the initial tumour ( $44.3 \pm 3.1 \%$ positive nuclei) versus (c) the recurrent tumour (17.7 $\pm 3.8 \%$ positive nuclei). (b) PMS2 staining in the initial tumour $(92.3 \pm 3.5 \%$ positive nuclei) versus (d) the recurrent tumour ( $28.3 \pm 6.9 \%$ positive nuclei). (B) The percentage of MLH1 and PMS2 immunopositive cells in each clinical initial and recurrent gliobastoma specimens is shown (paired $t$-test, $P<0.01)$. 
$(19.7 \pm 14.8 ; 45.3 \pm 27.2 \%)$ in comparison to their respective initial tumours $(46.9 \pm 19.1 ; 88.0 \pm 7.4 \%)(p$ $<0.01$, paired $t$-test) (Fig. 3B). By contrast, there was no correlation between the attenuation of MGMT expression and recurrence (Supplementary Figure S3A, B).

\section{DISCUSSION}

Previous studies have suggested that MGMT and base excision repair (BER) are involved in TMZ resistance $[15,16]$. MGMT also repairs the $\mathrm{O}^{6}$-chloroethylguanine residues induced by SN2-type methylating agents such as ACNU [17, 18]. Additionally, BER is involved in MMS resistance [19]. However, our TMZ-resistant cells did not show cross-resistance to such methylating agents (Table1). In addition, the $M G M T$ promoter was methylated in both the TMZ-resistant cells as well as the parental U251 cells (Supplementary Figure S1C). These results suggest that MGMT and BER are not involved in the acquisition of TMZ resistance. Furthermore, in clinical samples, we could not find any correlation between MGMT expression and tumour recurrence (Supplementary Figure S3A, B).

Previous studies have indicated a relationship between deficiencies in MMR components (MSH6, MSH2, MLH1, PMS2) and GBM recurrence [5-7]; however it remains unclear which component is most relevant for the acquisition of TMZ resistance. A decrease in MLH1 expression was observed in all three independent TMZ-resistant cell lines, and attenuation of MLH1 expression consistently modulated TMZ sensitivity in several GBM cell lines (Fig. 1A, B; Table 2). Furthermore, PMS2 protein expression was reduced in these TMZresistant cells and was correlated to that of MLH1 (Fig. 1A, B).

We clearly demonstrated that MLH1 or PMS2 knockdown confers TMZ resistance to GBM cells. Interestingly, the protein expression of PMS2 was not correlated with its mRNA expression levels. MLH1 knockdown by siRNA decreased PMS2 protein expression and not PMS2 mRNA expression. Moreover, the expression of PMS2 protein was associated with that of MLH1 protein in several GBM cell lines (Fig. 2A, B). However, the protein expression of $\mathrm{MSH} 2$ and beta-catenin was not affected by attenuation of MLH1 expression (Fig. 1D). This would suggest that MLH1 protein expression specifically affects the stability of the PMS2 protein. In contrast, PMS2 knockdown slightly affected the protein expression of MLH1 (Fig. 2C). Previously, Mohd et al. showed that PMS2 was stabilized in the presence of MLH1 through heterodimer formation by using an overexpression system [20]. We obtained consistent results indicating that MLH1 expression is responsible for the stability of endogenous PMS2 protein in GBM cells using MLH1 siRNA.

Stark et al. have demonstrated that MLH1 expression is significantly reduced in recurrent GBM, and that its expression in initial lesions was an indicator of reduced patient survival [6]. On the other hand, Flesberg et al. showed that the expression of MSH6, MSH2, and PMS2 were reduced in recurrent GBM, but that MLH1 expression was not correlated to recurrence [7]. However, in the Felsberg study, $32.6 \%$ of the recurrent tumours showed lower levels of MLH1 expression than in the primary tumours. This could mean that a decrease in MutL alpha may occur. We revealed that the suppression of MLH1 expression decreases the expression of PMS2 protein in TMZ-resistant cells and GBM cell lines in vitro. However, in our clinical data, though the expression of MLH1 and PMS2 decreases, the alternation of the expression of these genes did not always correlate each other (Fig. 3B). In addition, the protein expression of MSH6 was also decreased in U251/TMZR1 cells (Fig. 1B). These facts suggest that a decrease in MLH1 expression occurs early during the acquisition of TMZ resistance and, accordingly, the expression of PMS2 protein is reduced, although other factors may influence the expression of MMR component. However, the mechanism by which this occurs remains to be elucidated.

There is some new knowledge about the mechanism of chemoresistance in GBM. Urszula et al. revealed that loss of PDCD4 contributes to enhanced chemoresistance in GBM [21]. Swapna et al. revealed that epigenetic regulation of miRNA-211 by MMP-9 give the insensitivity of GBM to radiation and TMZ [22]. It might necessary to examine the function of such molecules in our TMZresistant cells. In addition, some studies propose new drugs for GBM treatment $[23,24]$. Some clues to overcome TMZ resistance might be revealed by examine the effect of such new drugs against our TMZ-resistance cells.

In summary, we have shown that U251 GBM cells acquire resistance to TMZ by reducing MLH1 expression following destabilization of PMS2 protein that is attenuated by MutL alpha. In addition, a significant reduction in MLH1 and PMS2 expression was observed in recurrent GBM tumours during TMZ administration. Our data suggest that a reduction in MLH1 protein expression leads to PMS2 protein instability, which confers TMZ resistance on GBM cells. This could lead to the recurrence of GBM during the course of TMZ treatment. Evaluating the expression of MLH1 and PMS2 in GBM may therefore provide a useful index for predicting the efficiency of TMZ anti-tumour activity.

\section{MATERIALS AND METHODS}

\section{Drugs, Reagents and antibodies}

The following reagents were purchased from the indicated companies (in brackets): RPMI 1640 
(Nikken Biomedical Laboratory, Osaka, Japan); Dulbecco's modified Eagle's medium (DMEM) (Nissui Seiyaku, Tokyo, Japan); foetal calf serum (FCS) (PAA Laboratories, Pasching, Austria); Coulter DNA Prep Reagents Kit (Beckman Coulter, Inc, Fullerton, CA, USA); Ac-DEVD-MCA (Ac-Asp-Glu-Val-Asp-MCA) (Wako Pure Chemical Industries, Osaka, Japan); MTT (3-(4,5-dimethylthiazol-2-yl)-2,5-diphenyl tetrazolium bromide) (Sigma-Aldrich, St, Louis, MO, USA); SYBR ${ }^{\circledR}$ Premix Ex Taq ${ }^{\text {TM }}$ II (Takara, Osaka, Japan); monoclonal antibodies against MLH1, beta-catenin, MGMT, MSH2, GAPDH (glyceraldehyde-3-phosphate dehydrogenase), MSH6, PMS2 and alpha-tubulin (BD Pharmingen, San Diego, CA,USA; Chemicon International, Inc, Temecula, CA, USA; Cell Signaling Technology, Inc., Danvers, MA, USA; Abcam, Cambridge, UK; and Calbiochem, San Diego, CA, USA, respectively).

\section{Cells and cell culture}

Human U251, U373, U105, LN229 GBM cells were cultured in RPMI 1640 containing 10\% foetal calf serum and a $1 \%$ antibiotic-antimycotic solution (Invitrogen, Carlsbad, CA, USA). To isolate TMZ-resistant cells, U251 cells were cultured in selection medium containing 400 micro-M TMZ and cloned by using the limiting dilution method. Three TMZ-resistant cells were then isolated and named U251/TMZR1, U251/TMZR2 and U251/ TMZR3. All cell lines were cultured at $37{ }^{\circ} \mathrm{C}$ in a $5 \% \mathrm{CO}_{2}-$ humidified atmosphere.

\section{RNA interference}

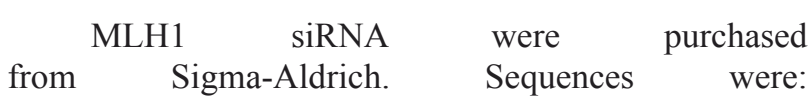
5'-UCACAAGUAUUCAAGUGAdTdT-3'(Sense oligo\# 7134001) and 5'-UCACUUGAAUACUUGUGGAdTdT3'(Antisense oligo\#7134002). The PMS2 siRNA duplexes were based on the coding region of the gene of interest, designed to contain dTdT overhangs, and were obtained from FASMAC. The sequences of the siRNAs were: 5'-CAAUGUUACUCCAGAUAAAdTdT-3'(Sense) and 5'-UUUAUCUGGAGUAACAUUGdTdT-3'(Antisense). Silencer $^{\circledR}$ Negative Control No. 1 siRNA (Ambion, Catalog \#: AM4611) was used as the control. One day before transfection, cells were seeded into $6-\mathrm{cm}$ tissue culture dishes at a density of $3 \times 10^{5}$ cells. Cells were then transfected with siRNA using Lipofectamine 2000 (Invitrogen, Carlsbad, CA, USA) according to the manufacturer's instructions. The cells were transferred to 96-well plates $\left(5 \times 10^{2} /\right.$ well $) 24 \mathrm{~h}$ after transfection for assay using the MTT colorimetric assay, or were transferred to 6-cm tissue culture dishes $48 \mathrm{~h}$ after transfection for realtime PCR and immunoblotting analyses.

\section{MTT assay of cell survival}

Equal numbers of cells $\left(5 \times 10^{2}\right)$ were inoculated into each well and the cells were treated for 7 days with TMZ, ACNU, MMS, or MNNG before the sensitivity of the cells to each of the administered drugs was measured using a MTT colorimetric assay as described previously [25].

\section{Caspase- 3 activation assay}

U251 and U251/TMZR2 cells were seeded into a 6 -cm tissue culture dish at a density of $2 \times 10^{5}$ cells. After treatment with 800 micro-M TMZ for $96 \mathrm{~h}$, the cells were trypsinized, and caspase-3 activation assays were carried out as described previously [26].

\section{Measurement of apoptotic cells and cell-cycle analysis by flow cytometry}

U251 or U251/TMZR2 cells were seeded into 6 -cm dishes at a density of $2 \times 10^{4}$ cells. After overnight incubation, the cells were treated with 800 micro-M TMZ for 24, 48 or $120 \mathrm{~h}$. Measurements of apoptotic cells and cell-cycle analysis were carried out by flow cytometry as described previously [27].

\section{Methylation-specific PCR.}

See Supplementary Materials and Methods.

\section{RNA isolation and cDNA synthesis}

Total RNA from the cultured cells was isolated using the TRI reagent (Molecular Research Center, Cincinnati, $\mathrm{OH}, \mathrm{USA}$ ) according to the manufacturer's instructions. RNA (1 micro-g) was reverse-transcribed using the ReverTra Ace kit (Toyobo, Osaka, Japan).

\section{Reverse transcription-PCR}

See Supplementary Materials and Methods.

\section{Quantitative real-time PCR.}

The mRNA expression levels of MSH6, MSH2, MLH1 and PMS2 were determined by real-time RTPCR (PRISM 7900HT; Applied Biosystems, Foster City, CA, USA) using SYBR ${ }^{\circledR}$ Premix Ex Taq ${ }^{\mathrm{TM}}$ II (Takara, Osaka, Japan) according to the manufacturer's instructions. Human GAPDH was used for normalization. The expression of the target gene was quantified by using the comparative cycle threshold method. Forward 
and reverse primers respectively were as follows: for MSH6, 5'- AGAGCAATGCAACGTGCAGA3' and 5'- TTTGGCGGCTACTTCGCCTA-3'; for MSH2, 5'- TTTACCCGGAGGAGAGACTGC3' and 5'- TGCTCTCCCTTTTTGCCTTTC-3'; for MLH1, p5'- TGTGCTGGCAATCAAGGGAC3' and 5'- TGTCCACGGTTGAGGCATTG-3'; for PMS2, 5'- ATCGGCGAAGGTTGGAACTC-3' and 5'- CGGATGCCTGCTGAAATGAT-3'; for GAPDH, 5'- TGCACCACCAACTGCTTAG-3' and 5'GAGGCAGGGATGATGTTC-3'.

\section{Protein extraction and immunoblotting}

The cells were harvested and lysed with RIPA buffer (25 mM Tris- $\mathrm{HCl}(\mathrm{pH} 7.5), 150 \mathrm{mM} \mathrm{NaCl}$, 1\% Nonidet P-40 (NP-40), 0.1\% SDS, 0.5\% sodium deoxycholate, $1 \mathrm{mM}$ p-amidinophenyl methanesulfonyl fluoride hydrochloride (APMSF), and $1 \mathrm{micro}-\mathrm{g} / \mathrm{mL}$ aprotinin), passed through a 27-gauge needle, and centrifuged at $14000 \mathrm{~g}$ for $15 \mathrm{~min}$ at $4{ }^{\circ} \mathrm{C}$. The supernatant was immediately used or was stored at $-80^{\circ} \mathrm{C}$ until use. Protein concentrations were determined using the BioRad protein assay kit. Cell lysates (100 micro-g protein) were subjected to $7.4 \%$ or $9.4 \%$ SDS-polyacrylamide gel electrophoresis (SDS-PAGE) and transferred onto membranes as described previously [26]. The blotted membranes were incubated with anti-MGMT (1:1000 dilution), anti-MLH1 (1:2000 dilution), anti-PMS2 (1:1000 dilution), anti-MSH6 (1:500 dilution), anti-MSH2 (1:1000 dilution), anti-beta-catenin (1:1000 dilution) or anti-GAPDH (1:200000 dilution) antibody overnight at 4 ${ }^{\circ} \mathrm{C}$, and each protein was detected as described previously [26].

\section{Immunohistochemical analysis of patient tumours}

Tumour specimens were obtained from nine GBM and two anaplastic astrocytoma (AA) patients. After the first surgery, all patients were treated with TMZ $\left(75 \mathrm{mg} / \mathrm{m}^{2}\right.$ daily for 42 days) concurrent with conventional radiation therapy (60 Gy), followed by TMZ $\left(200 \mathrm{mg} / \mathrm{m}^{2}\right)$ every 28 days according to the EORTC/NCIC-protocol [3] at the Department of Neurosurgery, Kagoshima University Hospital.

Surgically obtained specimens were fixed in $10 \%$ formaldehyde and then embedded in paraffin before being cut into 3 micro-m slices. Microwave antigen retrieval was performed in citrate buffer $(\mathrm{pH} \mathrm{6.0)}$ before the samples were incubated with the MLH1 mouse monoclonal antibody (1:100 dilution), PMS2 rabbit monoclonal antibody (1:200 dilution), or the MGMT mouse monoclonal antibody (1:100 dilution) as the primary antibody. The number of cells in three microscopic fields (magnification $\times 400$ ) was counted independently by two researchers (Y.S and S.Y). Ratios of the positive cells were obtained by dividing the number of immunopositive cells by the total number of cells per field, and are expressed as a percentage. Approval for this study was obtained from the Clinical Study Review Board of Kagoshima University Medical and Dental Hospital.

\section{Statistical analysis}

Statistical comparisons were performed using student's $t$ test. For immunohistochemical analysis, statistical comparisons were performed using the paired $t$ test. Quantitative data were expressed as the means \pm SD. $\mathrm{P}<0.05$ were considered significant.

\section{ACKNOWLEDGEMENTS}

The authors thank Ms. Hiromi Mitsuo for her excellent secretarial assistance and Ms. Mai Tokudome for performing the immunostaining. This work was supported by Grant-in-Aid for Scientific Research from the Ministry of Education, Culture, Sports, Science, and Technology of Japan.

\section{Funding:}

Grant-in-Aid for Scientific Research from the Ministry of Education, Culture, Sports, Science, and Technology of Japan.

\section{Conflict of interest:}

The authors have no conflicts of interest to disclose.

\section{REFERENCES}

1. Cohen MH, Johnson JR and Pazdur R. Food and Drug Administration Drug approval summary: temozolomide plus radiation therapy for the treatment of newly diagnosed glioblastoma multiforme. Clinical cancer research : an official journal of the American Association for Cancer Research. 2005; 11(19 Pt 1):6767-6771.

2. Stupp R, Dietrich PY, Ostermann Kraljevic S, Pica A, Maillard I, Maeder P, Meuli R, Janzer R, Pizzolato G, Miralbell R, Porchet F, Regli L, de Tribolet N, Mirimanoff RO and Leyvraz S. Promising survival for patients with newly diagnosed glioblastoma multiforme treated with concomitant radiation plus temozolomide followed by adjuvant temozolomide. Journal of clinical oncology : official journal of the American Society of Clinical Oncology. 2002; 20(5):1375-1382.

3. Stupp R, Mason WP, van den Bent MJ, Weller M, Fisher B, Taphoorn MJ, Belanger K, Brandes AA, Marosi C, Bogdahn U, Curschmann J, Janzer RC, Ludwin SK, 
Gorlia T, Allgeier A, Lacombe D, et al. Radiotherapy plus concomitant and adjuvant temozolomide for glioblastoma. The New England journal of medicine. 2005; 352(10):987996.

4. Schofield MJ and Hsieh P. DNA mismatch repair: molecular mechanisms and biological function. Annual review of microbiology. 2003; 57:579-608.

5. Cahill DP, Levine KK, Betensky RA, Codd PJ, Romany CA, Reavie LB, Batchelor TT, Futreal PA, Stratton MR, Curry WT, Iafrate AJ and Louis DN. Loss of the mismatch repair protein MSH6 in human glioblastomas is associated with tumor progression during temozolomide treatment. Clinical cancer research : an official journal of the American Association for Cancer Research. 2007; 13(7):2038-2045.

6. Stark AM, Doukas A, Hugo HH and Mehdorn HM. The expression of mismatch repair proteins MLH1, MSH2 and MSH6 correlates with the Ki67 proliferation index and survival in patients with recurrent glioblastoma. Neurological research. 2010; 32(8):816-820.

7. Felsberg J, Thon N, Eigenbrod S, Hentschel B, Sabel MC, Westphal M, Schackert G, Kreth FW, Pietsch T, Loffler M, Weller M, Reifenberger G, Tonn JC and German Glioma N. Promoter methylation and expression of MGMT and the DNA mismatch repair genes MLH1, MSH2, MSH6 and PMS2 in paired primary and recurrent glioblastomas. International journal of cancer Journal international du cancer. 2011; 129(3):659-670.

8. Hegi ME, Diserens AC, Gorlia T, Hamou MF, de Tribolet N, Weller M, Kros JM, Hainfellner JA, Mason W, Mariani L, Bromberg JE, Hau P, Mirimanoff RO, Cairncross JG, Janzer RC and Stupp R. MGMT gene silencing and benefit from temozolomide in glioblastoma. The New England journal of medicine. 2005; 352(10):997-1003.

9. Kaina B, Christmann M, Naumann S and Roos WP. MGMT: key node in the battle against genotoxicity, carcinogenicity and apoptosis induced by alkylating agents. DNA repair. 2007; 6(8):1079-1099.

10. Cejka P, Stojic L, Mojas N, Russell AM, Heinimann K, Cannavo E, di Pietro M, Marra G and Jiricny J. Methylationinduced $\mathrm{G}(2) / \mathrm{M}$ arrest requires a full complement of the mismatch repair protein hMLH1. The EMBO journal. 2003; 22(9):2245-2254.

11. O'Brien V and Brown R. Signalling cell cycle arrest and cell death through the MMR System. Carcinogenesis. 2006; 27(4):682-692.

12. Roos WP, Batista LF, Naumann SC, Wick W, Weller M, Menck CF and Kaina B. Apoptosis in malignant glioma cells triggered by the temozolomide-induced DNA lesion O6-methylguanine. Oncogene. 2007; 26(2):186-197.

13. Stojic L, Mojas N, Cejka P, Di Pietro M, Ferrari S, Marra $\mathrm{G}$ and Jiricny J. Mismatch repair-dependent G2 checkpoint induced by low doses of SN1 type methylating agents requires the ATR kinase. Genes \& development. 2004; 18(11):1331-1344.
14. Wang JY and Edelmann W. Mismatch repair proteins as sensors of alkylation DNA damage. Cancer cell. 2006; 9(6):417-418.

15. Brandes AA, Franceschi E, Tosoni A, Bartolini S, Bacci A, Agati R, Ghimenton C, Turazzi S, Talacchi A, Skrap M, Marucci G, Volpin L, Morandi L, Pizzolitto S, Gardiman M, Andreoli A, et al. O(6)-methylguanine DNAmethyltransferase methylation status can change between first surgery for newly diagnosed glioblastoma and second surgery for recurrence: clinical implications. Neurooncology. 2010; 12(3):283-288.

16. Bobola MS, Kolstoe DD, Blank A, Chamberlain MC and Silber JR. Repair of 3-methyladenine and abasic sites by base excision repair mediates glioblastoma resistance to temozolomide. Frontiers in oncology. 2012; 2:176.

17. Esteller M, Garcia-Foncillas J, Andion E, Goodman SN, Hidalgo OF, Vanaclocha V, Baylin SB and Herman JG. Inactivation of the DNA-repair gene MGMT and the clinical response of gliomas to alkylating agents. The New England journal of medicine. 2000; 343(19):1350-1354.

18. Pegg AE. Mammalian O6-alkylguanine-DNA alkyltransferase: regulation and importance in response to alkylating carcinogenic and therapeutic agents. Cancer research. 1990; 50(19):6119-6129.

19. Kundu CN, Balusu R, Jaiswal AS and Narayan S. Adenomatous polyposis coli-mediated hypersensitivity of mouse embryonic fibroblast cell lines to methylmethane sulfonate treatment: implication of base excision repair pathways. Carcinogenesis. 2007; 28(10):2089-2095.

20. Mohd AB, Palama B, Nelson SE, Tomer G, Nguyen M, Huo $\mathrm{X}$ and Buermeyer AB. Truncation of the C-terminus of human MLH1 blocks intracellular stabilization of PMS2 and disrupts DNA mismatch repair. DNA repair. 2006; 5(3):347-361.

21. Liwak U, Jordan LE, Von-Holt SD, Singh P, Hanson JE, Lorimer IA, Roncaroli F and Holcik M. Loss of PDCD4 contributes to enhanced chemoresistance in Glioblastoma Multiforme through de-repression of Bcl-xL translation. Oncotarget. 2013.

22. Asuthkar S, Velpula KK, Chetty C, Gorantla B and Rao JS. Epigenetic regulation of miRNA-211 by MMP-9 governs glioma cell apoptosis, chemosensitivity and radiosensitivity. Oncotarget. 2012; 3(11):1439-1454.

23. Triscott J, Lee C, Hu K, Fotovati A, Berns R, Pambid M, Luk M, Kast RE, Kong E, Toyota E, Yip S, Toyota B and Dunn SE. Disulfiram, a drug widely used to control alcoholism, suppresses the self-renewal of glioblastoma and over-rides resistance to temozolomide. Oncotarget. 2012; 3(10):1112-1123.

24. Kast RE, Boockvar JA, Bruning A, Cappello F, Chang WW, Cvek B, Dou QP, Duenas-Gonzalez A, Efferth T, Focosi D, Ghaffari SH, Karpel-Massler G, Ketola K, Khoshnevisan A, Keizman D, Magne N, et al. A conceptually new treatment approach for relapsed glioblastoma: coordinated undermining of survival paths with nine repurposed drugs 
(CUSP9) by the International Initiative for Accelerated Improvement of Glioblastoma Care. Oncotarget. 2013; 4(4):502-530.

25. Chen ZS, Aoki S, Komatsu M, Ueda K, Sumizawa T, Furukawa T, Okumura H, Ren XQ, Belinsky MG, Lee K, Kruh GD, Kobayashi M and Akiyama S. Reversal of drug resistance mediated by multidrug resistance protein (MRP) 1 by dual effects of agosterol A on MRP1 function. International journal of cancer Journal international du cancer. 2001; 93(1):107-113.

26. Che XF, Zheng CL, Owatari S, Mutoh M, Gotanda T, Jeung HC, Furukawa T, Ikeda R, Yamamoto M, Haraguchi M, Arima $\mathrm{N}$ and Akiyama S. Overexpression of survivin in primary ATL cells and sodium arsenite induces apoptosis by down-regulating survivin expression in ATL cell lines. Blood. 2006; 107(12):4880-4887.

27. Ikeda R, Furukawa T, Kitazono M, Ishitsuka K, Okumura H, Tani A, Sumizawa T, Haraguchi M, Komatsu M, Uchimiya $\mathrm{H}$, Ren XQ, Motoya $\mathrm{T}$, Yamada $\mathrm{K}$ and Akiyama S. Molecular basis for the inhibition of hypoxiainduced apoptosis by 2-deoxy-D-ribose. Biochemical and biophysical research communications. 2002; 291(4):806812. 